\title{
Validity and Reliability of the Interaction Rating Scale between Children (IRSC) by Using Motion Capture Analysis of Head Movement
}

\author{
Anme T. ${ }^{1, *}$, Sugisawa Y. ${ }^{1}$, Shinohara R. ${ }^{2}$, Matsumoto M. ${ }^{1}$, Watanabe T. ${ }^{1}$, Tokutake K. ${ }^{1}$, Tomisaki E. ${ }^{1}$, \\ Mochizuki H. ${ }^{1}$, Tanaka E. ${ }^{1}$, Okazaki S. ${ }^{3}$, Koeda T. ${ }^{4}$, Sadato N. ${ }^{3}$ \\ ${ }^{1}$ Faculty of Medicine, University of Tsukuba, Ibaragi, Japan \\ ${ }^{2}$ Yamanash University, Yamanashi, Japan \\ ${ }^{3}$ National Institute for Physiological Sciences, Aichi, Japan \\ ${ }^{4}$ Faculty of Education, Tottori University, Tottori, Japan
}

\begin{abstract}
The aim of this paper was to validate the Interaction Rating Scale for Children (IRSC) as an evidence-based practical index of social skills. Twenty 5-6-year-old preschool students participated in our study. Participants were required to complete a 5-minute interaction session and were evaluated using the IRSC. A motion capture machine, based on an ecologically valid interaction within a candy-distribution setting, monitored participants' head movements. Results indicated a moderately high correlation between IRSC scores and head movements. When cooperation was high, the "Pitch" was higher $(\mathrm{r}=0.548, \mathrm{p}<.05)$ and the "Yaw" was lower $(\mathrm{r}=-0.685, \mathrm{p}<.01)$. When self-control was high, the "Pitch" was lower $(\mathrm{r}=-0.576, \mathrm{p}<.01)$. The IRSC is able to measure social competence with high validity. Thus, the IRSC is a helpful tool for understanding the development of peer relationships.
\end{abstract}

Keywords Interaction,Peer, Children, Evaluation, Scale

\section{Introduction}

Peer interactions provide opportunities for children to learn social skills from each other and understand rules for appropriate behaviours in different settings. Moreover, social relationships established through interactions are a major source of feelings of security and belonging[1].

Social competence is defined as the ability to understand others in the context of a social interaction and engage in smooth communication with others. Thus, social competence should be evaluated by the interaction between a person and their social environment[2]. However, methodologies that consider people in conjunction with their social environment across developmental stages have not yet been well developed.

Early interaction is focused on measuring the quality of a child's home environment and parenting, based on the theory that an early rearing environment is significantly related to child development. Two instruments, namely, the Home Observation for Measurement of the Environment (HOME)[3] and the Index of Child Care Environment (ICCE)[4], are often used in research on child development.

* Corresponding author:

anmet@md.tsukuba.ac.jp (Anme T.)

Published online at http://journal.sapub.org/phr

Copyright (C) 2012 Scientific \& Academic Publishing. All Rights Reserved
The Interaction Rating Scale (IRS) was developed to measure a child's social skills and the caregiver's child rearing skills through observations of caregiver-child interactions, based on the HOME[3], SSRS (Social Skills Rating Systems)[5], and NCAST (Nursing Child Assessment Satellite Training) teaching scales[6](Sumner $\&$ Spietz, 1994). The IRS contains 70 items for a behavioral score and 11 items for an impression score, grouped into 10 subscales (5 subscales focus on children's social skills: Autonomy, Responsiveness, Empathy, Motor regulation, and Emotional regulation; another 5 items assess caregiver's parenting skills: Respect for autonomy development, Respect for responsiveness development, Respect for empathy development, Respect for cognitive development, and Respect for social-emotional development). This measure is used to evaluate, in less than 5 minutes, an observation appropriate for the assessment of interactions between caregivers and children from birth to 8 years old. The reliability of the IRS is 0.91 , and its validity with the NCAST is $r=.89$ [7-9].

The Interaction Rating Scale Advanced (IRSA)[10] was targeted to evaluate interactions for children over 15 years old and was developed from several sources. The study authors created some of the original items, and several overlapping items were taken from the IRS (Interaction Rating Scale)[7-9], the SSRS (Social Skills Rating Systems)[5], and the ENDCOREs[11]. The IRSA includes 
92 items that form a behavioral score and 6 subscales that comprise an impression score, which are "self-control," "expressivity," "sensitivity," "assertiveness," "responsiveness ," and "regulation." The reliability of IRSA is 0.84 and its validity with a practical evaluation is $\mathrm{r}=.65$.

Several studies assessing social competence have included common factors related to "empathy/cooperation," "self-control," and "assertion." These three factors have been found to be stable from the ages of 1 to 6 among Japanese children in one longitudinal study[9].

It is essential to develop methods to evaluate social skills relevant to peer relationships among children for the promotion of healthy development.

The purpose of this study was to clarify the validity and reliability of the Interaction Rating Scale between Children (IRSC: see Appendix 1) as an evidence-based practical index of social competence.

\section{Methods}

\subsection{Participants}

Participants were children enrolled in a longitudinal study of social development ("Sukusuku cohort" in Tottori group). Data were obtained from 20 children (5-year-olds: 8 boys and 4 girls, 6-year-olds: 8 boys) whose head movements could be measured.

In order to comply with ethical standards prior to conducting the research, all participants signed informed consent forms and were made aware that they had the right to withdraw from the experiment at any time. To maintain confidentiality of personal information among participants, a personal ID system was used. Furthermore, all image data were stored on a disk, which was password protected; only researchers who were granted permission had access to the data.

The ethics committee of the Research Institute of Science and Technology for Society approved this study.

\subsection{Measures}

The IRSC was used to assess social competence of children. The IRSC was developed as a peer relationship version of the IRS[7-9], which evaluates child-caregiver interactions by observations available for children under the age of 8 . The IRSC is used to measure social competence through a 5-minute observation of interactions. This includes three subscales measuring cooperation (20 items), self-control (10 items), and assertion (13 item; See Appendix 1). Scores for all IRSC subscales are derived from laboratory-based observations of the interactions of 4 children. IRSC items are based on items from the Interaction Rating Scale (IRS: for mother-child interactions)[7-9]. We also referred to the Interaction Rating Scale Advanced (IRSA; for adolescence over)[10].

In previous studies, social competence from infancy to adolescence had been classified into three core dimensions[5, 12, and 13]. The three core dimensions of social competence (cooperation, self-control, and assertion) have been described repeatedly in previous studies.

Two different sets of variables are scored in the IRSC: behavior items and impression items for each subscale. Each subscale assesses the presence of a behavior $(1=$ Yes, $0=$ No), and the sum of all items in the subscale provides the overall behavior score.

Scores on the impression items, and the overall impression item, are rated on a 5-point scale, where $1=$ not evident at all, $2=$ not clearly evident, $3=$ neutral, $4=$ evident, and $5=$ evident at high level.

The evaluator completes the checklist composed of 43 items focusing on behavior (e.g., Expresses his/her opinion well with words). Then the evaluator provides an impression on a 5-point scale of the level of interaction for each subscale.

Internal consistency of the IRSC, as measured by Cronbach's alpha, is 0.87 . The inter-observer reliability was $90 \%$. The IRSC can evaluate the interactions in a short period of time in daily situations.

A motion capture machine evaluated head movements. To decide the facing direction of the child's head, two markers (markers at the center and the anterior part of a cap) were selected, and this vector was calculated using a self-produced MATLAB program. We also calculated vertical and horizontal rotations of this vector as pitching and yawing motions, respectively. In addition, time profiles of the pitch and yaw head motions were estimated by multivariate autoregressive (MAR) models, and their Akaike's noise contribution ratio (NCR) was calculated. The NCR revealed the causality of children's head motion from its past and the history of the head motion in the other 3 children, individually[14]. For the MAR model estimation, decimation, window length, window-shift length, and max order of the AR model were set at $3(30->10 \mathrm{~Hz}), 10$ seconds, 1 second, and 20, respectively. Averaged noise contribution ratios during the experiment from the self and the other 3 children were assessed for associations with the IRSC.

\subsection{Procedure}

For the current study, a 5-minute video recording the interaction setting was used to evaluate the IRSC. Four participants distributed candy in an ecologically valid environment. The recording was carried out in a room with 4 video cameras. The participants were escorted into a room furnished with a small table. The instructor asked participants to distribute the candies.

Two evaluators coded the participant's behaviors. The behavior during the interaction was coded as follows. If the participant displayed the behavior described in the item, a score of 1 was given. Conversely, if the participant failed to display the behavior described in the item, a score of 0 was given. Total scores were the sum of the score that participants received on all the subscales. A higher score indicated a higher level of social competence. The total IRSC score was the combined score totaled from each subscale. 
Table 1. Summary statistics on social competence

\begin{tabular}{cccccccc} 
Subscale & $\mathrm{N}$ & mean & median & mode & S.D. & minimum & maximum \\
\hline Cooperation & 20 & 13.10 & 13.5 & 15 & 2.10 & 8 & 15 \\
Self-Control & 20 & 9.20 & 10 & 10 & 1.58 & 4 & 10 \\
Assertion & 20 & 9.55 & 10 & 10 & 2.16 & 5 & 12 \\
\hline Total score & 20 & 31.85 & 33.5 & 36 & 4.53 & 20 & 39 \\
\hline
\end{tabular}

Table 2. Social competence and head movement

\begin{tabular}{cccccc}
\hline & & \multicolumn{5}{c}{ IRSC score } \\
\cline { 3 - 6 } & & Cooperation & Self-Control & Assertion & Total \\
\hline \multirow{2}{*}{ Pitch } & $\mathrm{r}$ & 0.548 & -0.576 & 0.333 & 0.358 \\
& $\mathrm{p}$ & 0.012 & 0.008 & 0.151 & 0.121 \\
\hline \multirow{2}{*}{ Yaw } & $\mathrm{r}$ & -0.685 & -0.056 & -0.239 & -0.491 \\
& $\mathrm{p}$ & 0.001 & 0.815 & 0.310 & 0.028 \\
\hline
\end{tabular}

\subsection{Analysis}

Correlation analysis was conducted to examine the relationship between head movement and social competence in 5- and 6-year-olds. Spearman's correlation coefficient was used to evaluate the correlation at a significance level of $\mathrm{p}<.05$. The Statistical Analysis System (version 9.1) was used for data analysis.

\section{Results}

We examined the relationship between children's social competence and head movement in the candy distribution setting.

Table 1 shows the summary statistics of social competence using the IRSC.

Table 2 shows the correlation between IRSC scores and head movements.

When cooperation was high, the "Pitch" was higher $(\mathrm{r}=$ $0.548, \mathrm{p}<.05)$, and the "Yaw" was lower $(\mathrm{r}=-0.685$, $\mathrm{p}$ $<.01)$. When self-control was high, the "Pitch" was lower $(\mathrm{r}$ $=-0.576, \mathrm{p}<.01)$.

\section{Discussion}

The current study suggests that the IRSC can measure social competence with adequate validity and reliability. Results indicated a moderately high correlation between the IRSC and children's head movements. In an ecologically valid situation, the "pitch" was used as a "yes" or "I hear you talking" response. However, the "yaw" seemed to indicate denial behavior and was not often used to indicate cooperation. In addition, the "pitch" is not often used to indicate self-control. This is considered a reasonable result with respect to the assessment of social competence in our candy distribution scenario.

Social interaction is an important context that serves to mediate the links between cultural values and individual development. Social competence in peer interactions plays a crucial role in building links between the individual and his/her culture[15]. During interactions, peers evaluate individual behaviors in ways that are consistent with norms endorsed by their peer world[16]. Peers react to these behaviors accordingly by expressing acceptance or rejection toward children who display certain behaviors. Social competence may regulate children's behaviors and, ultimately, their developmental trajectory. We examined social competence between children who were focused on specific behaviors, which we expected to reflect cultural and social norms[17].

Social competence is determined by the complex interaction between the individual, their home and school environments, peer relationships, and the larger sociocultural environment[18]. Increasing numbers of school-aged children and adolescents displaying impulsive behaviors and maladjustment to society necessitate societal preparedness for appropriate education and environments to foster pro-social behaviors among these youth.

The social competence scale for child-caregiver interaction, referred to as the Interaction Rating Scale (IRS), and for adult interactions, referred to as the Interaction Rating Scale Advanced (IRSA), were already found to be a reliable, valid, feasible, and practical tools in studies assessing social interactions over time[7-10].

Three strengths of the IRSC are described below.

First, the IRSC is easy to use in practice, because it is highly adaptable where the subscale framework can be used across several age groups.

Second, the IRSC can be used in international comparative studies, because it is based on the most common frameworks used all over the world. The subscales are based on various categories, which are widely used in research on social competence indicators.

Third, we have evidence of the IRSC's construct and concurrent validity via our motion capture analysis of head movements.

While the IRSC provides valuable insights, it is also important to acknowledge its limitations. First, we present results from a small pilot study; only 20 participants were in our study. The generalizability of our results is tenuous. Second, the IRSC subscales might not cover all the dimensions of social competence, even though we used the most common frameworks of social competence. Third, 
while the IRSC uses the same scoring standard as a standardized tool, different developmental features of certain items across developmental stages might need to be assessed further. Despite these limitations, the IRSC can be considered an established, valid screening instrument reflecting attributes of social competence. This measure helps provide further evidence of the development of social competence, including several features of social interactions among the IRSC subscales.

\section{Conclusions}

This studyprovides evidence that children's peer relationships can be evaluated with validity and reliability. Further research has the potential to reveal features of problems with the development of social competence, such as issues among children with attention-deficit hyperactivity disorder. Such information should enhance knowledge of implications for practitioners and caregivers.

\section{ACKNOWLEDGEMENTS}

This research was supported by Grant-in-Aid for Scientific Research to N.S. (S21220005) from the Japan Society for the Promotion of Science. Part of this study is the result of the project "Development of biomarker candidates for social behavior" carried out under the Strategic Research Program for Brain Sciences, by the Ministry of Education, Culture, Sports, Science and Technology (MEXT) and Grants-in-Aid for Scientific Research (23330174, 24653134).

\section{Appendix 1 Interaction Rating Scale for School Children (IRSC)}

\section{Cooperation: children's cooperative and empathetic behaviors}

1) Does not vocalize, make a facial expression, or move in response to the partner's gestures after the partner's utterance.

2) Babbles, makes a facial expression, or moves in response to the partner's behavior or nonverbal cues.

3) Does not vocalize, make a facial expression, or move in response to the partner's behavior or nonverbal cues.

4) Smiles in response to the partner's smile.

5) Smiles, claps hands, or shows he/she is glad when the partner is feeling happy.

6) Shares intentions and attitudes with the partner through eye contact.

7) Praises the partner's efforts, success, and behavior.

8) Smiles and/or nods at the partner during the conversation.

9) Talks to the partner positively or encouragingly during the assignment.
10) Does not vocalize or interrupt the partner while he/she is speaking.

11) Accepts the partner's opinion partially or totally by saying, "let's do it" or by acting in a manner consistent with the partner's suggestion.

12) Vocalizes and/or moves in response to the partner's appearance after the partner's utterance.

13) Says a word of thanks, such as "thank you" to the partner.

14) Says a word of apology such as "I am sorry" to the partner.

15) Gives a soothing non-verbal response (i.e., pat, touch, or rock).

16) Shows empathy with a verbal or non-verbal response when the partner is in a bad mood.

17) Vocalizes and/or moves in a humorous manner to brighten the atmosphere.

18) Does not criticize the partner.

19) Tries to respond appropriately during confrontation with the partner.

20) Tries to respond calmly when the partner becomes angry or agitated.

\section{Self-Control: children's behaviors that emerge in conflict situations}

21) Is not rude to the partner and not destructive/rough with the materials.

22) Does not disturb the partner.

23) Concentrates on the task and is gentle with the materials.

24) Does not lose his/her temper.

25) Attempts to elicit help or consolation from the partner.

26) Does not display distress cues even when the task does not go well.

27) Does not tell a lie and does not cheat.

28) Does not speak negatively of others and the materials.

29) Follows the rules of the tasks.

30) Behaves in accordance with the expectations of others.

\section{Assertion: children's initiating behaviors}

31) Expresses his/her opinion well with words.

32) Shows his/her feelings well through gestures and behavior.

33) Smiles or laughs.

34) Looks at the partner's face to get information/clarification.

35) Turns his/her gaze to the partner when trying to get sympathy.

36) Shows his/her feelings well with his/her facial expressions.

37) Speaks up to the partner about what he/she thinks.

38) The partner responds to his/her proposal or decision. 
39) Exhibits a differing opinion with his/her expression and gestures.

40) Verbalizes a differing opinion or position.

41) Provides guidance through explanation and not through order.

42) Explains his/her opinion based on the level of competence/ability of the partner.

43) Explains his/her opinion logically.

- Harmony and adaptation: Being in harmony with the partner (having adapted to the group).

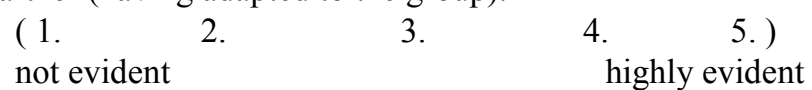

-Influence: Having initiative.
( 1 . 2.
3.
4. 5. )

not evident highly evident

-Group cohesiveness: Having a dynamic relationship with the partner (in the group).
(1.
2.3 .
not evident
$4 . \quad 5$.
highly evident

[7] Anme T, Yato Y, Shinohara R, Sugisawa Y. The reliability and validity of the assessment method for children's social competence: Parent-child Interaction Rating Scale. Japanese Journal of Human Science of Health-Social Services, 14, 23-31, 2007.

[8] Anme T. Trajectories of social competence by using Interaction Rating Scale (IRS) as an evidence-based practical index of children's social skills and parenting. Journal of Epidemiology, 20: 419-426. 2010.

[9] Anme T. Gender differences of children's social skills and parenting using Interaction Rating Scale (IRS). Procedia Social and Behavioural Sciences, 2: 260-268, 2010.

[10] Anme T, Watanabe T, Tokutake K. A pilot study of social competence assessment using Interaction Rating Scale Advanced (IRSA). ISRN Pediatrics. doi:10.5402/2011/ 2729132011

[11] Fujimoto M, Daibo K. ENDCOREs: A Hierarchical Structure Theory of Communication Skills. The Japanese Journal of Personality, 15: 347-361. 2007.

[12] Caldarella P, Merrell KW. Common dimensions of social skills of children and adolescents: taxonomy of positive behaviors. School Psychology Review, 26, 264-278. 1997.

[13] Elksnin LK,Elksnin N. Teaching social skills to students with learning and behavior problems. Intervention in School \& Clinic, 33(3), 131-141. 1998.

[14] Bosch-Bayard J, Wong, KFK, Okazaki S, Oshio R, Galka A, Ozaki T, Sadato N. Directed Causality for Non-stationary Time Series Based on Akaike's Noise Contribution Ratio. Formath, 11, 121-131, 2012.

[15] Henirch CC, Blatt SJ, Kuperminc GP, Zohar A, Leadbeater BJ. Levels of interpersonal concerns and socialfunctioning in early adolescent boys and girls. Journal ofPersonality Assessment, 76, 48-67, 2001.

[16] Keller H, Yovsi R, Borke J, Kartner J, Jensen H, Papaligoura Z. Developmental consequences of early parenting experiences: Self-recognition and self-regulation in three culturalcommunities. Child Development, 75, 1745-1760, 2004.

[17] Kolb SM, Hanley-Maxwell C. Critical social skills for adolescents with high incidence disabilities: parental perspectives. Exceptional Children,69(2), 163-179. 2003.

[18] Riggio ER. Assessment of Basic Social Skills. Journal of Personality and Social Psychology 51(3): 649-660. 1986.

[6] Barnard K. Nursing Child Assessment Satellite Training Manual. 1994. 